\title{
Gasteditorial des Präsidenten der Leibniz-Gemeinschaft, Prof. Ernst Th. Rietschel
}

\author{
Ernst Th. Rietschel
}

Online publiziert: 19. März 2010

(C) Springer-Verlag 2010

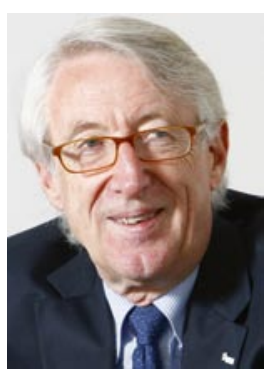

(Bildnachweis: Ralf Günther)

Publikationen in wissenschaftlichen Fachzeitschriften sind ein wesentlicher Qualitätsindikator für Forschungsleistungen. Sicherlich ist dieser Umstand in den Lebens- und Naturwissenschaften am stärksten ausgeprägt, wo die Impact-Faktoren eminente Bedeutung erlangt haben, indem sie über die relative Menge von Zitaten pro Artikel die wissenschaftliche Reputation einer Zeitschrift widerspiegeln. In anderen Disziplinen wie den Sozial- und Geisteswissenschaften ist - zurzeit allerdings noch deutlich moderater - eine ähnliche Entwicklung zu beobachten.

Gleichwohl gilt für alle Wissenschaftsdisziplinen, dass der fachliche Austausch über Fachpublikationen ein ganz wesentlicher Bestandteil der Kommunikation innerhalb der jeweiligen „scientific community“ ist. „Raumforschung und Raumordnung" ist ein solches renommiertes Organ mit einer durchaus bewegten Geschichte, aber fast die einzige referierte Zeitschrift ihres Fachgebiets im deutschsprachigen

Prof. Dr. Dr. h.c. E. Th. Rietschel $(\bowtie)$

Präsident der Leibniz-Gemeinschaft,

Schützenstraße 6a,

10117 Berlin, Deutschland

E-Mail: praesident@leibniz-gemeinschaft.de
Raum. Im 68. Jahrgang wird nun ein neues Kapitel in dieser Geschichte aufgeschlagen.

Mit dem Wechsel im Herausgeberkreis wird diese wichtige interdisziplinäre raumwissenschaftliche Zeitschrift quasi zu einem „Leibniz-Produkt": Neben die bekannte Alt-Herausgeberin, die Akademie für Raumforschung und Landesplanung (ARL) aus Hannover, sind nun weitere raumwissenschaftliche Institute der Leibniz-Gemeinschaft getreten: das Leibniz-Institut für Länderkunde (IfL) in Leipzig, das Leibniz-Institut für ökologische Raumentwicklung (IÖR) in Dresden und das Leibniz-Institut für Regionalentwicklung und Strukturplanung (IRS) in Erkner. Außerdem wirkt mit dem ILS - Institut für Landes- und Stadtentwicklungsforschung $\mathrm{gGmbH}$ aus Dortmund auch ein assoziiertes Mitglied der Leibniz-Gemeinschaft an der Produktion der Zeitschrift mit.

Die nicht-universitäre wie die universitäre raumbezogene Diskussion und Forschung hat in „Raumforschung und Raumordnung" eine Plattform für Grundlagendiskurse ebenso wie für Fragen der Anwendungsorientierung. Gemeinsam ist diesen Fragen, dass sie sich theoretisch, methodisch oder im Blick auf die Umsetzung und Implementierung von Erkenntnis mit der Lösung der großen, die Gesellschaft bewegenden Fragen im Rückgriff auf Ergebnisse der Wissenschaft beschäftigten - auch das ist übrigens typisch für die Mitglieder der Leibniz-Gemeinschaft insgesamt.

Die raumwissenschaftlichen Leibniz-Einrichtungen haben früh erkannt, wo die Zukunft für die Leibniz-Gemeinschaft als Wissenschaftsorganisation liegt: in der Bildung themenbezogener Verbünde, wo unterschiedliche Untersuchungsansätze, fachliche Expertisen und Erfahrungs-KnowHow gebündelt und flexibel kombiniert wird.

Raumwissenschaftliche Forschung ist im Kern zukunftsbezogene Forschung. Weltweit steigen die Ansprüche, Kon- 
flikte und Probleme in Verbindung mit der nachhaltigen Nutzung des Raumes und der natürlichen Lebensgrundlagen. Die Dynamik der Globalisierung und des wirtschaftlichen Strukturwandels nimmt zu, die europäische Integration sowie die Transformationsprozesse in Mittelund Osteuropa sind verstärkt auf der politischen Agenda. Die Folgen dieser Prozesse sind weit reichende räumliche Veränderungen. In den Städten und Regionen wachsen die Gegensätze. All diese Entwicklungen haben unmittelbare Auswirkungen auf die Gesellschaft, auf unseren Alltag. Sie betreffen die wirtschaftlichen und sozialen Lebensbedingungen und die natürlichen Grundlagen aller Menschen. Die Forschungen aller raumwissenschaftlichen Einrichtungen der Leibniz-Gemeinschaft beschäftigen sich vor dem Hintergrund des komplexen Wechselspiels von Mensch und Raum mit der Frage, wie hier eine zukunftsfähige Entwicklung möglich ist.

Die Einrichtungen der Leibniz-Gemeinschaft sehen es als Bestandteil ihres Wissenschaftsverständnisses an, die gewonnenen Forschungsergebnisse für die Gesellschaft nutzbar zu machen. Dieser Wissenstransfer richtet sich in unterschiedlicher Form an die eigene oder an benachbarte wissenschaftliche Fachdisziplinen, als Politik- oder Gesellschaftsberatung an Entscheidungsträger in Parlamenten, Verwaltungen und Wirtschaft sowie an die allgemeine Öffentlichkeit. Somit stellt das Engagement der fünf raumwissenschaftlichen Einrichtungen der Leibniz-Gemeinschaft eine konsequente Verwirklichung unseres Wissenschaftsverständnisses dar, das wir unter dem Motto Gottfried Wilhelm Leibniz' „theoria cum praxi“ als „Wissenschaft zum Nutzen und Wohl der Menschen" verstehen.

Ich bin sehr zuversichtlich, dass der raumbezogenen Forschung und der Praxis mit ,Raumforschung und Raumordnung“" auch in Zukunft ein hochwertiges Organ in bewährter Qualität zur Verfügung steht, in dem zuverlässig zukunftsweisende Fragestellungen diskutiert und neue, innovative Themenfelder abgesteckt werden.

In diesem Sinne wünsche ich allen Leserinnen und Lesern eine erkenntnisreiche und Ideen fördernde Lektüre und allen an der Herausgabe Beteiligten eine erfolgreiche Kooperation.

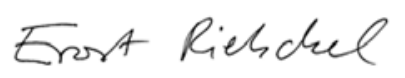

